\title{
Measurement of IR optics with linear coupling's action-angle parametrization
}

\author{
Y. Luo, ${ }^{*}$ M. Bai, F. Pilat, T. Satogata, and D. Trbojevic \\ Brookhaven National Laboratory, Upton, New York 11973, USA \\ (Received 31 March 2005; published 10 August 2005; corrected 12 August 2005)
}

\begin{abstract}
Linear coupling's action-angle parametrization is convenient for interpretation of turn-by-turn beam position monitor (BPM) data. We demonstrate how to apply this parametrization to extract Twiss and coupling parameters in interaction regions (IRs), using BPMs on each side of a long IR drift region. Example data were acquired at the Relativistic Heavy Ion Collider, using an ac dipole to excite a single transverse eigenmode. We have measured the waist of the $\beta$ function and its Twiss and coupling parameters.
\end{abstract}

DOI: 10.1103/PhysRevSTAB.8.084001

PACS numbers: 29.20.Dh, 29.27.Bd

\section{INTRODUCTION}

Measurement of $\beta^{*}$ at the collision point plays an important role in collider operations. The conventional method of measuring $\beta^{*}$ through knobbing the first focusing quadrupole next to the interaction point does not provide adequate resolution. Another method of measuring the second order tune shift with $\pm \Delta k$ changes to the first two focusing quadrupoles only applies to the colliders with symmetric IR optics. And its key parameter, $\alpha_{\text {optics }}$, comes from off-line optics simulation. These two methods assume that the optics is not coupled [1].

Linear coupling's action-angle parametrization is a strict treatment for linearly coupled optics [2-6]. It is convenient for analytical calculation and for interpretation of turn-byturn (TBT) beam position monitor (BPM) data. In this article, we demonstrate how to apply it to extract Twiss and coupling parameters in an interaction region (IR). In most colliders, there is a BPM in either side of interaction region (IR) drift, so the TBT $\left(x, x^{\prime}, y, y^{\prime}\right)$ data in the IR drift can be obtained. The propagation of optics parameters in the IR drift is also derived to determine the optics at the beta waist. This approach is tested with the TBT BPM data taken under a single transverse eigenmode excitation with ac dipole at the Relativistic Heavy Ion Collider (RHIC).

\section{BACKGROUND}

In this section, the linear coupling's action-angle parametrization is briefly reviewed. We give interpretation of TBT BPM data of single eigenmode excitation and provide expressions for IR optics propagation.

\section{A. Action-angle parametrization}

For the general two-dimensional linearly coupled motion, a single particle's motion is represented as [2]

$$
\left(\begin{array}{c}
x \\
x^{\prime} \\
y \\
y^{\prime}
\end{array}\right)=\mathbf{P}\left(\begin{array}{c}
\sqrt{2 J_{\mathrm{I}}} \cos \Phi_{\mathrm{I}} \\
-\sqrt{2 J_{\mathrm{I}}} \sin \Phi_{\mathrm{I}} \\
\sqrt{2 J_{\mathrm{II}}} \cos \Phi_{\mathrm{II}} \\
-\sqrt{2 J_{\mathrm{II}}} \sin \Phi_{\mathrm{II}}
\end{array}\right),
$$

where $J_{\mathrm{I}, \mathrm{II}}$ and $\Phi_{\mathrm{I}, \mathrm{II}}$ are the actions and phases of the two eigenmodes. The eigentunes are $\mu_{\mathrm{I}}$ and $\mu_{\mathrm{II}} \cdot \phi_{\mathrm{I}, 0}$ and $\phi_{\mathrm{II}, 0}$ are the eigenmodes' initial phases. They can be calculated from

$$
\begin{aligned}
& \left\{\begin{array}{l}
\phi_{\mathrm{I}, 0}=\arctan \left(\frac{-S_{\mathrm{I}}}{C_{\mathrm{I}}}\right), \\
C_{\mathrm{I}}=\sum_{i=1}^{N} x_{i} \cos \left[2 \pi \mu_{\mathrm{I}}(n-1)\right], \\
S_{\mathrm{I}}=\sum_{i=1}^{N} x_{i} \sin \left[2 \pi \mu_{\mathrm{I}}(n-1)\right],
\end{array}\right. \\
& \left\{\begin{array}{l}
\phi_{\mathrm{II}, 0}=\arctan \left(\frac{-S_{\mathrm{II}}}{C_{\mathrm{II}}}\right), \\
C_{\mathrm{II}}=\sum_{i=1}^{N} y_{i} \cos \left[2 \pi \mu_{\mathrm{II}}(n-1)\right], \\
S_{\mathrm{II}}=\sum_{i=1}^{N} y_{i} \sin \left[2 \pi \mu_{\mathrm{II}}(n-1)\right],
\end{array}\right.
\end{aligned}
$$

where $N$ is the maximum turn for the calculation. Equations (2) and (3) hold for large $N$. According to the eigenmode phase definitions, $p_{12}=p_{34}=0$.

$\mathbf{P}$ can be numerically determined from the eigenvectors of the one-turn $4 \times 4$ transfer map, or from Twiss and coupling parameters defined in Edwards-Teng's parametrization,

$$
\mathbf{P}=\left(\begin{array}{cccc}
r \sqrt{\beta_{\mathrm{I}}} & 0 & c_{11} \sqrt{\beta_{I I}}-\frac{c_{12} \alpha_{I I}}{\sqrt{\beta_{\mathrm{II}}}} & \frac{c_{12}}{\sqrt{\beta_{\mathrm{II}}}} \\
-\frac{\alpha_{I} r}{\sqrt{\beta_{\mathrm{I}}}} & \frac{r}{\sqrt{\beta_{I}}} & c_{21} \sqrt{\beta_{\mathrm{II}}}-\frac{c_{22} \alpha_{\mathrm{II}}}{\sqrt{\beta_{\mathrm{II}}}} & \frac{c_{22}}{\sqrt{\beta_{I I}}} \\
-\frac{c_{12} \alpha_{\mathrm{I}}}{\sqrt{\beta_{\mathrm{I}}}}-c_{22} \sqrt{\beta_{I}} & \frac{c_{12}}{\sqrt{\beta_{\mathrm{I}}}} & r \sqrt{\beta_{\mathrm{II}}} & 0 \\
\frac{c_{11}}{\sqrt{\beta_{\mathrm{I}}}}+c_{21} \sqrt{\beta_{I}} & -\frac{c_{11}}{\sqrt{\beta_{\mathrm{I}}}} & -\frac{\alpha_{\mathrm{II}} r}{\sqrt{\beta_{\mathrm{II}}}} & \frac{r}{\sqrt{\beta_{\mathrm{II}}}}
\end{array}\right) .
$$

\footnotetext{
*Electronic address: yluo@bnl.gov
} 


$$
\begin{gathered}
\left\{\begin{array}{l}
\beta_{\mathrm{I}}=\frac{p_{11}}{p_{22}} \\
\alpha_{\mathrm{I}}=-\frac{p_{21}}{p_{22}}, \\
\gamma_{\mathrm{I}}=\frac{1+\alpha_{1}^{2}}{\beta_{\mathrm{I}}}=\frac{p_{21}^{2}+p_{22}^{2}}{p_{11} p_{22}},
\end{array}\right. \\
\left\{\begin{array}{l}
\beta_{\mathrm{II}}=\frac{p_{33}}{p_{44}} \\
\alpha_{\mathrm{II}}=-\frac{p_{43}}{p_{44}}, \\
\gamma_{\mathrm{II}}=\frac{1+\alpha_{\mathrm{II}}^{2}}{\beta_{\mathrm{II}}}=\frac{p_{43}^{2}+p_{44}^{2}}{p_{33} p_{44}}, \\
r=\sqrt{p_{11} p_{22}}=\sqrt{p_{33} p_{44}},
\end{array}\right. \\
\mathbf{C}=r \mathbf{P}_{12} \mathbf{P}_{22}^{-1}=-\frac{1-r^{2}}{r} \mathbf{P}_{11} \mathbf{P}_{21}^{-1} .
\end{gathered}
$$

\section{B. Single eigenmode excitation}

Single eigenmode excitation is used for the betatron optics measurement [7-10]. In RHIC, ac dipoles are used for this purpose [11]. For simplicity, Eq. (1) can be rewritten as

$$
\left(\begin{array}{c}
x \\
x^{\prime} \\
y \\
y^{\prime}
\end{array}\right)=\mathbf{F}\left(\begin{array}{c}
\cos \Phi_{\mathrm{I}} \\
-\sin \Phi_{\mathrm{I}} \\
\cos \Phi_{\mathrm{II}} \\
-\sin \Phi_{\mathrm{II}}
\end{array}\right),
$$

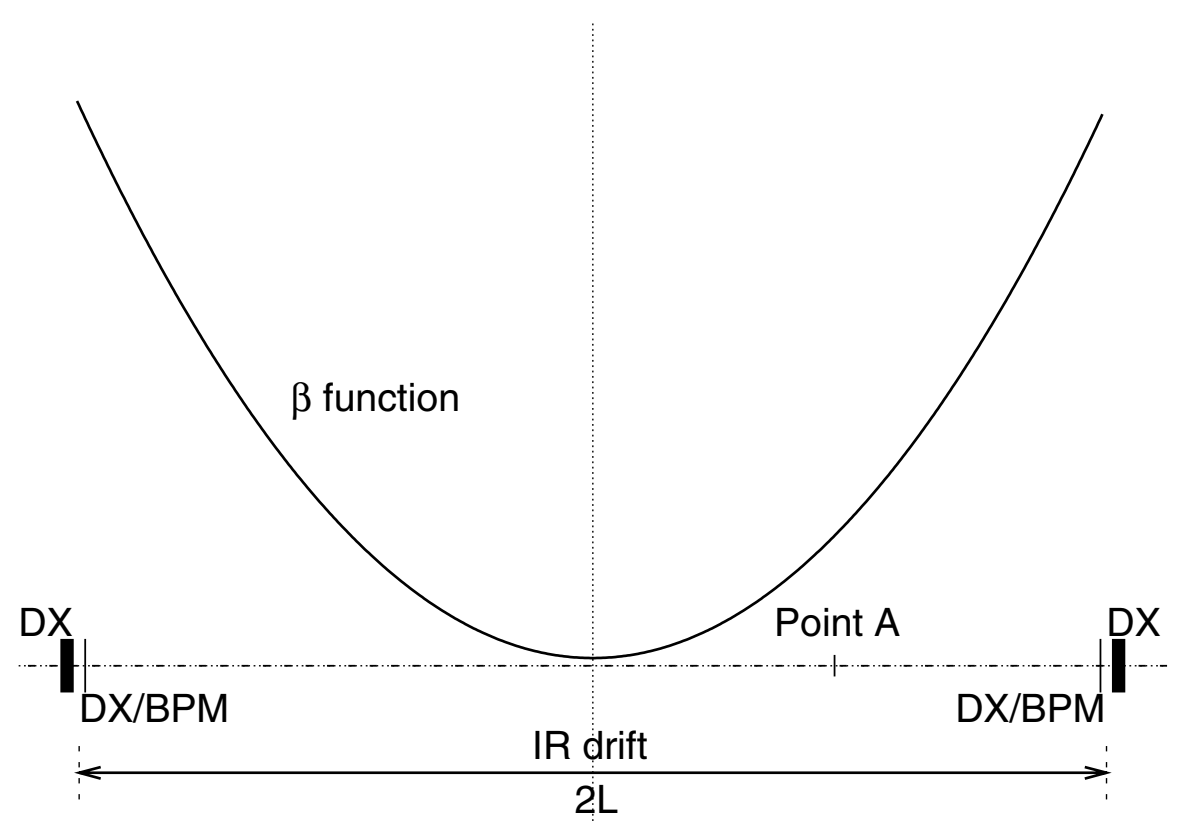

FIG. 1. IR drift between the two DX BPMs in one RHIC IR.

$$
\mathbf{F}=\left(\begin{array}{cccc}
p_{11} \sqrt{J_{\mathrm{I}}} & 0 & p_{13} \sqrt{J_{\mathrm{II}}} & p_{14} \sqrt{J_{\mathrm{II}}} \\
p_{21} \sqrt{J_{\mathrm{I}}} & p_{22} \sqrt{J_{\mathrm{I}}} & p_{23} \sqrt{J_{\mathrm{II}}} & p_{24} \sqrt{J_{\mathrm{II}}} \\
p_{31} \sqrt{J_{\mathrm{I}}} & p_{32} \sqrt{J_{\mathrm{I}}} & p_{33} \sqrt{J_{\mathrm{II}}} & 0 \\
p_{41} \sqrt{J_{\mathrm{I}}} & p_{42} \sqrt{J_{\mathrm{I}}} & p_{43} \sqrt{J_{\mathrm{II}}} & p_{44} \sqrt{J_{\mathrm{II}}}
\end{array}\right) .
$$

F can be determined from TBT $\left(x, x^{\prime}, y, y^{\prime}\right)$ data.

In this article, we assume that eigenmode $\mathrm{I}$ is more closely related to the horizontal plane than is eigenmode II, while eigenmode II is more associated with the vertical plane than eigenmode I. Thus, if only eigenmode I is excited, the elements in the last two columns of $\mathbf{F}$ are zero, and

$$
\begin{cases}p_{i, 1}=\frac{F_{i, 1}}{\sqrt{J_{\mathrm{I}}}}, & i=1,2,3,4 \\ p_{i, 2}=\frac{F_{i, 2}}{\sqrt{J_{\mathrm{I}}}}, & i=1,2,3,4\end{cases}
$$

\section{TBT $\left(x, x^{\prime}, y, y^{\prime}\right)$ data at DX BPMs}

In each IR of RHIC, there are two dual-plane BPMs that are close to the IR's DX separation magnets and face the interaction point, as shown in Fig. 1. These BPMs are called DX BPMs.

For RHIC, we ignore the effect of the detector solenoid field since its effect on the beam optics is small [12]. The TBT angles $\left(x^{\prime}, y^{\prime}\right)$ at the two DX BPMs can then be determined,

$$
\left\{\begin{array}{l}
x^{\prime}=\frac{x_{2}-x_{1}}{2 L}, \\
y^{\prime}=\frac{y_{2}-y_{1}}{2 L} .
\end{array}\right.
$$

$2 L$ is the distance between the two DX BPMs; for RHIC, $2 L=16.652 \mathrm{~m} .\left(x_{1}, y_{1}\right)$ are the BPM position readings at 
the upstream DX BPM, $\left(x_{2}, y_{2}\right)$ are the BPM position readings at the downstream DX BPM.

\section{Optics propagation in the IR drift}

At the $\beta$ waist, where $\alpha_{\mathrm{I}, \mathrm{II}}=0$,

$$
\mathbf{P}_{w}=\left(\begin{array}{cccc}
p_{w, 11} & 0 & p_{w, 13} & p_{w, 14} \\
0 & p_{w, 22} & p_{w, 23} & p_{w, 24} \\
p_{w, 31} & p_{w, 32} & p_{w, 33} & 0 \\
p_{w, 41} & p_{w, 42} & 0 & p_{w, 44}
\end{array}\right) .
$$

The subscript $w$ means the parameters are those at the $\beta$ waist. For simplicity, we assume that eigenmode I and eigenmode II's $\beta$ waists are located at the same point. Even if they are not, the following conclusions still hold. $\mathbf{T}_{w \rightarrow A}$ is the $4 \times 4$ drift transfer matrix from the waist to a point in the IR drift, say point A, as shown in Fig. $1 . l$ is the distance from the waist to point A.

Matrix $\mathbf{G}$ defined by Eq. (69) in [2] is

$$
\mathbf{G}=\mathbf{T}_{w \rightarrow A} \mathbf{P}_{w}=\left(\begin{array}{cccc}
p_{w, 11} & l p_{w, 22} & p_{w, 13}+l p_{w, 23} & p_{w, 14}+l p_{w, 24} \\
0 & p_{w, 22} & p_{w, 23} & p_{w, 24} \\
p_{w, 31}+l p_{w, 41} & p_{w, 32}+l p_{w, 42} & p_{w, 33} & l p_{w, 44} \\
p_{w, 41} & p_{w, 42} & 0 & p_{w, 44}
\end{array}\right)
$$

According to Eqs. (78) and (79) in [2], and considering Eqs. (5) and (6) at the $\beta$ waists, Twiss parameters at point A are obtained from Eq. (14),

$$
\begin{gathered}
\left\{\begin{array}{l}
\beta_{A, \mathrm{I}}=\beta_{w, \mathrm{I}}+\frac{l^{2}}{\beta_{w, \mathrm{I}}}, \\
\alpha_{A, \mathrm{I}}=-\frac{l}{\beta_{w, I}},
\end{array}\right. \\
\begin{cases}\beta_{A, \mathrm{II}}= & \beta_{w, \mathrm{II}}+\frac{l^{2}}{\beta_{w, \mathrm{II}}}, \\
\alpha_{A, \mathrm{II}} & =-\frac{l}{\beta_{w, \mathrm{II}}} .\end{cases}
\end{gathered}
$$

Subscript $A$ means the parameters are those at point A.

The location of $\beta$ waist and its $\beta$ value can then be obtained from measured Twiss parameters at point A,

$$
\begin{gathered}
\left\{\begin{array}{l}
\beta_{w, \mathrm{I}}=\frac{\beta_{A, \mathrm{I}}}{1+\alpha_{A, \mathrm{I}}^{2}} \\
\delta l_{w, \mathrm{I}}=-\alpha_{A, \mathrm{I}} \beta_{w, \mathrm{I}} \cdot
\end{array}\right. \\
\left\{\begin{array}{l}
\beta_{w, \mathrm{II}}=\frac{\beta_{A, \mathrm{II}}}{1+\alpha_{A, \mathrm{II}}^{2}} \\
\delta l_{w, \mathrm{II}}=-\alpha_{A, \mathrm{II}} \beta_{w, \mathrm{II}} .
\end{array}\right.
\end{gathered}
$$

$\delta l_{w, \mathrm{I}}$ and $\delta l_{w, \mathrm{II}}$ are the longitudinal offset of point A with respect to eigenmode I and eigenmode II's waists, respectively.

According to Eqs. (70) and (72) in [2], the phase advances from the $\beta$ waists to point $\mathrm{A}$ are calculated from Eq. (14),

$$
\begin{gathered}
\Delta \Phi_{\mathrm{I}}=\tan ^{-1}\left(\frac{\delta l_{w, \mathrm{I}}}{\beta_{w, \mathrm{I}}}\right), \\
\Delta \Phi_{\mathrm{II}}=\tan ^{-1}\left(\frac{\delta l_{w, \mathrm{II}}}{\beta_{w, \mathrm{II}}}\right) .
\end{gathered}
$$

Assuming that the $\beta$ waists occur at the center of the two DX BPMs, then the total phase advances between the two DX BPMs are

$$
\begin{gathered}
\left.\Delta \Phi_{\mathrm{I}}\right|_{d x b p m s}=2 \tan ^{-1}\left(\frac{L}{\beta_{c, I}}\right), \\
\left.\Delta \Phi_{\mathrm{II}}\right|_{d x b p m s}=2 \tan ^{-1}\left(\frac{L}{\beta_{c, \mathrm{II}}}\right),
\end{gathered}
$$

where $L$ is the distance from the IR center to the DX BPM, $\beta_{c} \mathrm{~s}$ are the $\beta$ functions at the IR center. The $\beta_{c} \mathrm{~s}$ at the IR center then can be determined from the phase advances between the two DX BPMs [10],

$$
\begin{gathered}
\beta_{c, \mathrm{I}}=\frac{L}{\tan \frac{\left.\Delta \Phi_{\mathrm{I}}\right|_{\text {dxbpms }}}{2}}, \\
\beta_{c, \mathrm{II}}=\frac{L}{\tan \frac{\left.\Delta \Phi_{\mathrm{III}}\right|_{x x b m s}}{2}} .
\end{gathered}
$$

Knowing the coupling matrix $\mathbf{C}$ at point $\mathrm{A}$ in the IR drift, according to Eq. (86) in [2], the coupling matrix $\mathbf{C}_{w}$ at the $\beta$ waist can be obtained,

$$
\begin{aligned}
& \mathbf{C}_{w}=\mathbf{M}_{d}^{-1} \mathbf{C}_{A} \mathbf{M}_{d}, \\
& \mathbf{M}_{d}=\left(\begin{array}{cc}
1 & \delta l_{w} \\
0 & 1
\end{array}\right) .
\end{aligned}
$$

$\delta l_{w}$ is the longitudinal offset of point $\mathrm{A}$ with respect to eigenmode I or eigenmode II's waist.

\section{MEASUREMENTS}

In the following, we demonstrate how to apply the above equations to extract the optics parameters in the IR drift. TBT BPM data used here was acquired with an ac dipole excitation at eigenmode I's fractional tune frequency.

We first calculate Twiss and coupling parameters at the blue ring IR6 center. The two dual-plane DX BPMs are rbpm.b-g5 and rbpm.b-g6. The blue beam circulates from rbpm.b-g5 to rbpm.b-g6. The TBT position data at the IR6 center are given by 


$$
\left\{\begin{array}{l}
x_{c}=\frac{x_{1}+x_{2}}{2}, \\
y_{c}=\frac{y_{1}+y_{2}}{2} .
\end{array}\right.
$$

The TBT angles $\left(x^{\prime}, y^{\prime}\right)$ at the IR6 center are calculated according to Eq. (12).

\section{A. Matrix F}

The eigentunes $\mu_{\mathrm{I}, \mathrm{II}}$ are obtained from fast Fourier transformation (FFT) of $(x+y)$. The initial phases $\phi_{\mathrm{I}, \mathrm{II}, 0}$ are calculated according to Eqs. (2) and (3). $F_{i j}$ s are obtained by

$$
\left\{\begin{array}{l}
F_{11}=\frac{2}{N} \sum_{i=1}^{N} x_{i} \cos \left[2 \pi \mu_{\mathrm{I}}(n-1)+\phi_{I, 0}\right] \\
F_{12}=-\frac{2}{N} \sum_{i=1}^{N} x_{i} \sin \left[2 \pi \mu_{\mathrm{I}}(n-1)+\phi_{I, 0}\right] \\
F_{21}=\frac{2}{N} \sum_{i=1}^{N} x_{i}^{\prime} \cos \left[2 \pi \mu_{\mathrm{I}}(n-1)+\phi_{\mathrm{I}, 0}\right] \\
F_{22}=-\frac{2}{N} \sum_{i=1}^{N} x_{i}^{\prime} \sin \left[2 \pi \mu_{\mathrm{I}}(n-1)+\phi_{\mathrm{I}, 0}\right] \\
F_{31}=\frac{2}{N} \sum_{i=1}^{N} y_{i} \cos \left[2 \pi \mu_{\mathrm{I}}(n-1)+\phi_{I, 0}\right] \\
F_{32}=-\frac{2}{N} \sum_{i=1}^{N} y_{i} \sin \left[2 \pi \mu_{\mathrm{I}}(n-1)+\phi_{I, 0}\right] \\
F_{41}=\frac{2}{N} \sum_{i=1}^{N} y_{i}^{\prime} \cos \left[2 \pi \mu_{\mathrm{I}}(n-1)+\phi_{\mathrm{I}, 0}\right] \\
F_{42}=-\frac{2}{N} \sum_{i=1}^{N} y_{i}^{\prime} \sin \left[2 \pi \mu_{\mathrm{I}}(n-1)+\phi_{\mathrm{I}, 0}\right]
\end{array}\right.
$$

$N=1024$ in this article. With the above TBT $\left(x, x^{\prime}, y, y^{\prime}\right)$ data at the blue ring IR6 center,

$$
\mathbf{F}=\left(\begin{array}{cccc}
1.24 \times 10^{-5} & 0 & 0 & 0 \\
-1.01 \times 10^{-6} & 1.27 \times 10^{-5} & 0 & 0 \\
-6.49 \times 10^{-7} & -9.74 \times 10^{-7} & 0 & 0 \\
-4.51 \times 10^{-7} & -9.29 \times 10^{-7} & 0 & 0
\end{array}\right)
$$

Measurement errors are briefly discussed in section IIIE. In the following, the measurement results are given to three significant figures.

\section{B. Optics parameters at IR center}

According to Eqs. (4), (11), and (29) at the IR6 center,

$$
\begin{gathered}
r \sqrt{J_{\mathrm{I}}}=\sqrt{F_{11} \bar{F}_{22}}=1.26 \times 10^{-5}\left[(\mathrm{~m} \mathrm{rad})^{-1 / 2}\right], \\
\frac{1-r^{2}}{r^{2}}=\frac{\left\|\mathbf{P}_{21}\right\|}{\left\|\mathbf{P}_{11}\right\|}=\frac{\left\|\mathbf{F}_{21}\right\|}{\left\|\mathbf{F}_{11}\right\|}=1.04 \times 10^{-2} .
\end{gathered}
$$

Therefore,

$$
\begin{gathered}
r=0.999, \\
\sqrt{J_{\mathrm{I}}}=1.26 \times 10^{-5}\left[(\mathrm{~m} \mathrm{rad})^{-1 / 2}\right] .
\end{gathered}
$$

From Eqs. (5) and (11), at the IR6 center,

$$
\left\{\begin{array}{l}
\beta_{c, I}=\frac{p_{11}}{p_{22}}=\frac{F_{11}}{F_{22}}=0.977[\mathrm{~m}], \\
\alpha_{c, I}=-\frac{p_{21}}{p_{22}}=-\frac{F_{21}}{F_{22}}=7.93 \times 10^{-2} .
\end{array}\right.
$$

According to Eqs. (8) and (11), the coupling matrix at the IR6 center is

$$
\mathbf{C}_{c}=\left(\begin{array}{cc}
7.30 \times 10^{-2} & -7.65 \times 10^{-2} \\
-4.22 \times 10^{-2} & 5.84 \times 10^{-2}
\end{array}\right)
$$

\section{Optics parameters at $\boldsymbol{\beta}$ waist}

Knowing eigenmode I's Twiss parameters at the IR6 center, according to Eq. (17), the $\beta$ waist in IR6 can be determined,

$$
\begin{gathered}
\beta_{w, \mathrm{I}}=0.971[\mathrm{~m}], \\
\delta l_{w, \mathrm{I}}=-7.70 \times 10^{-2}[\mathrm{~m}] .
\end{gathered}
$$

A negative $\delta l_{w, \mathrm{I}}$ means the $\beta_{\mathrm{I}}$ waist is sited downstream with respect to the IR center.

According to Eq. (25), the coupling matrix $\mathbf{C}_{w, \mathrm{I}}$ at eigenmode I's $\beta$ waist can be calculated,

$$
\mathbf{C}_{w, \mathrm{I}}=\left(\begin{array}{cc}
7.63 \times 10^{-2} & -7.52 \times 10^{-2} \\
-4.22 \times 10^{-2} & 5.51 \times 10^{-2}
\end{array}\right)
$$

\section{Summary of IR optics measurements}

Table I lists the measured Twiss and coupling at the IR centers. The coupling parameters are not available at IR2 center since the rbpm.b-g2 BPM vertical data are aberrant.

According to Eq. (23), Table II lists all IR centers' $\beta_{c, \mathrm{I}} \mathrm{S}$ calculated from the phase advances between the two adjacent DX BPMs.

Comparing $\beta_{c, \mathrm{I}}$ at the IR centers from Tables I and II, the difference at the IR12 center is apparent. Looking into the TBT BPM data of the IR12 DX BPMs, we found that the downstream BPM rbpm.b-g12 gave poor horizontal data.

Table III lists the locations of eigenmode I's waists and its optics parameters. $\delta l_{w, \mathrm{I}}$ is the longitudinal offset of the

TABLE I. Measured Twiss and coupling parameters at the IR centers.

\begin{tabular}{llccccc}
\hline \hline IRs & $\beta_{c, \mathrm{I}}$ & $\alpha_{c, \mathrm{I}}$ & $c_{11}$ & $c_{12}$ & $c_{21}$ & $c_{22}$ \\
\hline IR6 & 0.977 & $7.93 \times 10^{-2}$ & $7.30 \times 10^{-2}$ & $-7.65 \times 10^{-2}$ & $-4.22 \times 10^{-2}$ & $5.84 \times 10^{-2}$ \\
IR8 & 0.989 & $3.35 \times 10^{-2}$ & 0.255 & -1.71 & $-4.55 \times 10^{-2}$ & 1.03 \\
IR10 & 2.47 & $5.36 \times 10^{-2}$ & $-1.92 \times 10^{-2}$ & -0.495 & $9.10 \times 10^{-3}$ & $-2.13 \times 10^{-2}$ \\
IR12 & 3.28 & -0.393 & $4.84 \times 10^{-2}$ & -1.22 & $3.37 \times 10^{-2}$ & -0.277 \\
IR2 & 2.50 & 0.397 & & & & \\
IR4 & 4.89 & $-8.44 \times 10^{-2}$ & $-8.50 \times 10^{-2}$ & $3.83 \times 10^{-2}$ & $-3.44 \times 10^{-2}$ & -0.256 \\
\hline \hline
\end{tabular}


TABLE II. $\quad \beta_{\text {I }}$ at the IR centers calculated from the phase advances between DX BPMs.

\begin{tabular}{lllcccc}
\hline \multicolumn{1}{c}{ IRs } & IR6 & IR8 & IR10 & IR12 & IR2 & IR4 \\
\hline$\left.\Delta \Phi_{\mathrm{I}}\right|_{d x b p m s}[\mathrm{rad}]$ & 2.91 & 2.91 & 2.57 & 2.47 & 2.63 & 2.08 \\
$\beta_{c, \mathrm{I}}[\mathrm{m}]$ & 0.968 & 0.968 & 2.45 & 2.91 & 2.18 & 4.89 \\
\hline \hline
\end{tabular}

IR center with respect to the waist. IR2 and IR12 are excluded from Table III.

Table IV lists the coupling parameter $r$ and eigenmode I's action $\sqrt{J_{\mathrm{I}}}$ in the RHIC IRs. $\sqrt{J_{\mathrm{I}}}$ is a global constant. According to Table IV, the average of $\sqrt{J_{\mathrm{I}}}$ is

$$
\left\langle\sqrt{J_{\mathrm{I}}}\right\rangle=1.18 \times 10^{-5}\left[(\mathrm{~m} \mathrm{rad})^{-1 / 2}\right]
$$

\section{E. Measurement errors}

Linear coupling's action-angle parametrization is a strict treatment for linearly coupled optics. The errors in the above IR optics measurement come from the BPM readings and the data processing algorithms. It is not straightforward to estimate the measurement errors.
TABLE IV. $\quad r$ and $\sqrt{J_{\mathrm{I}}}$ in IRs.

\begin{tabular}{lcc}
\hline \hline IRs & $r$ & $\sqrt{J_{\mathrm{I}}}[\mathrm{m} \mathrm{mrad}]^{-1 / 2}$ \\
\hline IR6 & 0.999 & $1.26 \times 10^{-5}$ \\
IR8 & 0.911 & $1.17 \times 10^{-5}$ \\
IR10 & 0.997 & $1.11 \times 10^{-5}$ \\
IR4 & 0.988 & $1.09 \times 10^{-5}$ \\
\hline \hline
\end{tabular}

The RHIC BPM position resolution is $1 \mu \mathrm{m}$. However, the beam position accuracy is much worse than that. $10 \mathrm{~Hz}$ beam orbit oscillation from the $10 \mathrm{~Hz}$ helium flow was found [13]. The accuracy error for the RHIC BPM position readings is about $\pm 50 \mu \mathrm{m}$. Figure 2 shows the TBT $\left(x, x^{\prime}\right)$ at the IR6 center in the above measurement. How to reduce the RHIC BPM position reading errors is still under investigation.

Data processing algorithms are also important for the above measurement. The general FFT is used here to extract the eigentunes, and Eqs. (2), (3), and (28) only exactly hold with infinite $N$. To improve the tune estimate accuracy, the modified FFT algorithms should be used $[14,15]$, and robust TBT BPM data analysis methods, like principle component analysis (PCA) and independent

TABLE III. Optics parameters at $\beta_{\mathrm{I}}$ waist in IRs.

\begin{tabular}{lcccccc}
\hline \hline IRs & $\delta l_{w, \mathrm{I}}[\mathrm{m}]$ & $\beta_{w, \mathrm{I}}[\mathrm{m}]$ & $c_{11}$ & $c_{12}$ & $c_{21}$ & $c_{22}$ \\
\hline IR6 & $-7.70 \times 10^{-2}$ & 0.971 & $6.98 \times 10^{-2}$ & $-7.74 \times 10^{-2}$ & $-4.22 \times 10^{-2}$ & $6.16 \times 10^{-2}$ \\
IR8 & $-3.31 \times 10^{-2}$ & 0.987 & 0.278 & -1.85 & $-4.55 \times 10^{-2}$ & 1.03 \\
IR10 & -0.132 & 2.46 & $-1.80 \times 10^{-2}$ & -0.496 & $9.10 \times 10^{-3}$ & $-2.37 \times 10^{-2}$ \\
IR4 & 0.410 & 4.86 & $-7.22 \times 10^{-2}$ & 0.115 & $-3.48 \times 10^{-2}$ & -0.273 \\
\hline \hline
\end{tabular}

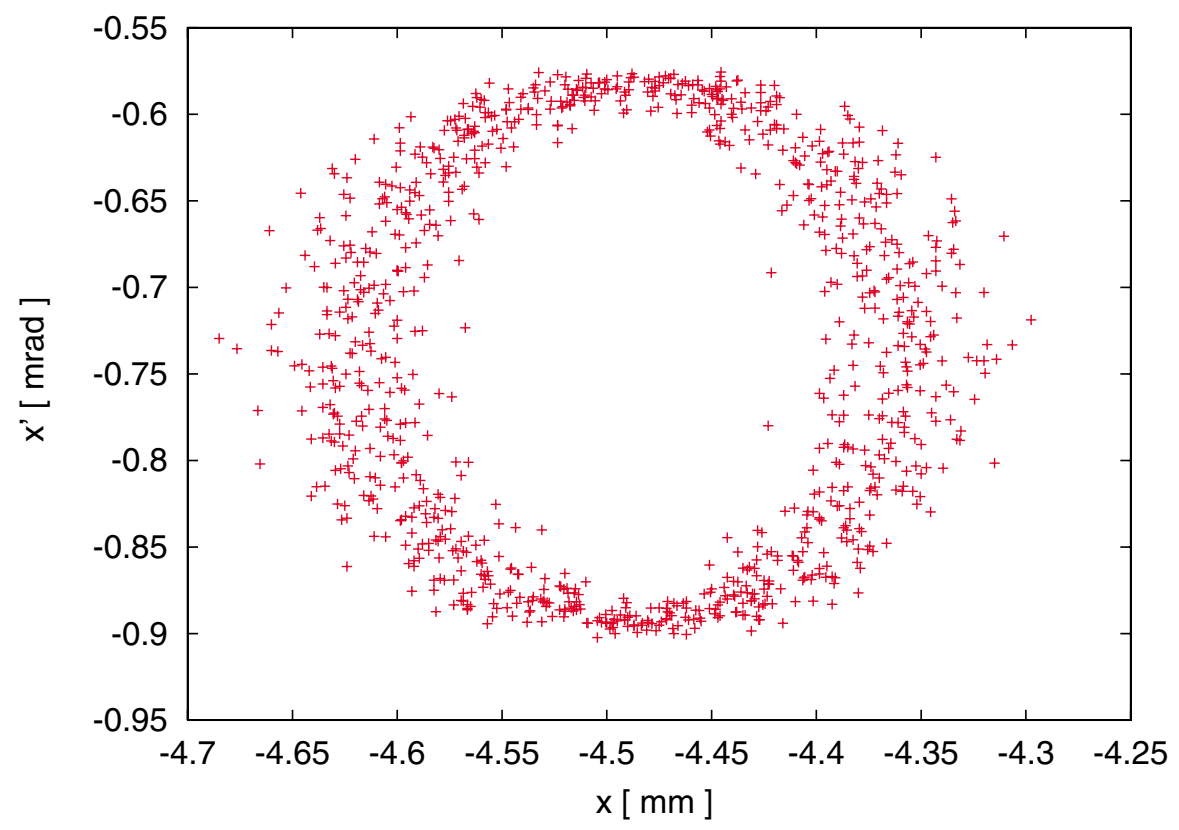

FIG. 2. (Color) Measured TBT (x, $\left.\mathrm{x}^{\prime}\right)$ at IR6 center. 
component analysis (ICA), can be applied to accurately determine the eigenmode motions and their phase advances $[16,17]$.

\section{CONCLUSION}

Linear coupling's action-angle parametrization has been tested for the IR optics measurement in the RHIC IRs. Based on the TBT BPM data at the two DX BPMs, the coupled optics parameters in the IR were determined. Measurement errors come from the BPM readings and the data processing algorithms, and are still under investigation. This method can be used to the optics measurements in any drift region with two dual-plane BPMs.

\section{ACKNOWLEDGMENTS}

Y. Luo would like to thank A. Woodhead for her help in preparing this manuscript. This work is support by the U.S. DOE under Contract No. DE-AC02-98CH10886.

[1] M.G. Minty and F. Zimmermann, Measurement and Control of Charged Beams (Springer, New York, 2003).

[2] Y. Luo, Phys. Rev. ST Accel. Beams 7, 124001 (2004).
[3] D. Sagan and D. Rubin, Phys. Rev. ST Accel. Beams 2, 074001 (1999).

[4] M. Billing, Cornell Report No. CBN 85-2, 1985.

[5] S. Peggs, Part. Accel. 12, 219 (1982).

[6] D. Edwards and L. Teng, IEEE Trans. Nucl. Sci. 20, 3 (1973).

[7] P. Bagley and D. Rubin, in Proceedings of the Particle Accelerator Conference, Chicago, IL, 1989 (IEEE, Piscataway, NJ, 1989), p. 874.

[8] D. Sagan, Phys. Rev. ST Accel. Beams 4, 122801 (2001).

[9] D. Sagan et al., Phys. Rev. ST Accel. Beams 3, 102801 (2000).

[10] Y. Cai, Phys. Rev. E 68, 036501 (2003).

[11] M. Bai, S. Peggs, T. Roser, T. Satogata, and D. Trbojevic, in Proceedings of the Particle Accelerator Conference, Portland, OR, 2003 (IEEE, Piscataway, NJ, 2003), p. 2204.

[12] T. Satogata, BNL CAD AP Note-19, 1993 (unpublished).

[13] C. Montag, G. Ganetis, L. Jia, and W. Louie, in Proceedings of the Particle Accelerator Conference, Portland, OR, 2003 (Ref. [11]), p. 1431.

[14] R. Bartolini, A. Bazzani, M. Giovannozzi, W. Scandale, and E. Todesco, CERN Report No. SL/95-84 (AP), 1995.

[15] J. Laskar, Physica (Amsterdam) 67D, 257 (1993).

[16] C.-x. Wang, V. Sajaev, and C.-y. Yao, Phys. Rev. ST Accel. Beams 6, 104001 (2003).

[17] X.-b. Huang, S. Y. Lee, E. Prebys, and R. Tomlin, Phys. Rev. ST Accel. Beams 8, 064001 (2005). 